\section{Predicting the surgical reparability of large-to-massive rotator cuff tears by B-mode ultrasonography: a cross-sectional study}

\author{
Po-Cheng Chen ${ }^{1,2}$, Kuan-Ting Wu ${ }^{3}$, Yi-Cun Chen ${ }^{1}$, Yu-Chi Huang ${ }^{1}$, Ching-Di Chang ${ }^{4}$, \\ Wei-Che Lin ${ }^{4}$, Wen-Yi Chou ${ }^{3,5}$ \\ * Author affiliations appear at the end of this article.
}

Purpose: This study aimed to compare the ability of B-mode ultrasonography and magnetic resonance imaging (MRI) to predict the repairability of large-to-massive rotator cuff tears (RCTs). Methods: This cross-sectional study included participants with large-to-massive RCTs who underwent arthroscopic repair. B-mode ultrasonography and MRI were conducted prior to arthroscopic repair. B-mode ultrasonography was used to evaluate the echogenicity of the rotator cuff muscle using the Heckmatt scale. Intra-rater and inter-rater reliabilities were examined for two independent physicians. MRI was used to evaluate the degrees of tendon retraction, fatty infiltration of rotator cuff muscles, and muscle atrophy. Finally, two experienced orthopedic surgeons performed surgery and decided whether the torn stump could be completely repaired intraoperatively.

Results: Fifty participants were included, and 32 complete repairs and 18 partial repairs were performed. B-mode ultrasonography showed good intra-rater reliability and inter-rater reliability for assessment of the muscle echogenicity of the supraspinatus and infraspinatus muscles. The correlation coefficients between B-mode ultrasound findings and MRI findings showed medium to large effect sizes $(r=0.4-0.8)$. The Goutallier classification of the infraspinatus muscles was the MRI predictor with the best discriminative power for surgical reparability (area under the curve $[A \cup C], 0.89 ; 95 \%$ confidence interval $[\mathrm{CI}], 0.81$ to 0.98 ), while the Heckmatt scale for infraspinatus muscles was the most accurate ultrasound predictor (AUC, $0.85 ; 95 \% \mathrm{Cl}, 0.74$ to 0.96). No significant differences in AUCs among the MRI and ultrasound predictors were found. Conclusion: B-mode ultrasonography was a reliable examination tool and had a similar ability to predict surgical reparability to that of MRI among patients with large-to-massive RCTs.

Keywords: Large-to-massive rotator cuff tears; Reparability; B-mode ultrasound

Key points: B-mode ultrasonography has comparable predictive ability of surgical reparability to that of MRI in patients with large-to-massive rotator cuff tears. B-mode ultrasonography would show good intra-rater and inter-rater reliabilities in assessment of echogenicity of rotater cuff muscles.

e-ultrasonography.org

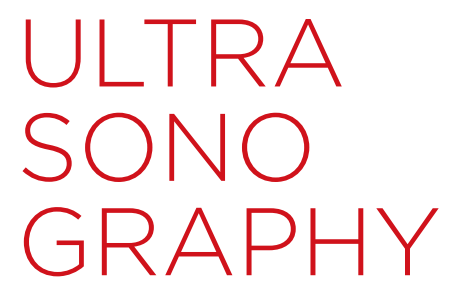

ORIGINAL ARTICLE

https://doi.org/10.14366/usg.20192 pISSN: 2288-5919 • elSSN: 2288-5943 Ultrasonography 2022;41:177-188

Received: December 2, 2020

Revised: June 4, 2021

Accepted: June 6, 2021

Correspondence to:

Wen-Yi Chou, MD, Department of Orthopedic Surgery, Kaohsiung Chang Gung Memorial Hospital, Chang Gung University College of Medicine, 123 Ta-Pei Road, Niao-Sung, Kaohsiung, Taiwan

Tel. +886-7-731-7123 (ext 8003)

Fax. +886-7-735-4309

E-mail: murraychou@adm.cgmh.org.tw

This is an Open Access article distributed under the terms of the Creative Commons Attribution NonCommercial License (http://creativecommons.org/ licenses/by-nc/4.0/) which permits unrestricted noncommercial use, distribution, and reproduction in any medium, provided the original work is properly cited.

Copyright (C) 2022 Korean Society of Ultrasound in Medicine (KSUM)

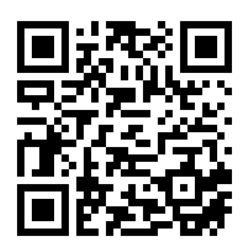

How to cite this article:

Chen PC, Wu KT, Chen YC, Huang YC, Chang $C D$, Lin WC, et al. Predicting the surgical reparability of large-to-massive rotator cuff tears by B-mode ultrasonography: a crosssectional study. Ultrasonography. 2022 Jan;41(1):177-188. 


\section{Introduction}

Rotator cuff tears (RCTs) usually result in shoulder pain and weakness in forward flexion and abduction to the horizontal position $[1,2]$. The prevalence of RCTs increases with age according to recent epidemiological studies $[3,4]$. A systematic review including a metaanalysis [5] reported that the dominant arm, being overweight, tobacco use, and hypertension were also risk factors for RCTs. Approximately $40 \%$ of all RCTs were found to be large-to-massive in an observational study [5]. In order to improve shoulder pain and shoulder function, most patients with symptomatic large-to-massive RCTs are willing to undergo surgical repair; however, the degree of rotator cuff muscle atrophy and fatty infiltration affects surgical reparability [6]. Large-to-massive RCTs can be repaired completely in some clinical situations, but are sometimes irreparable because of severe muscle atrophy or poor tissue quality. Whether or not the torn stump can be reduced to the anatomic footprint (complete repair) is evaluated by orthopedic surgeons intraoperatively. However, surgical reparability can also be predicted preoperatively with the assistance of some examinations.

Magnetic resonance imaging (MRI) was considered a suitable examination for predicting the surgical reparability of large-tomassive RCTs in previous studies [7-9], which evaluated tear size, tendon retraction, and fatty infiltration of the muscle layer by MRI. Despite the fact that MRI provides a detailed evaluation of the injured soft tissue, the clinical availability of MRI is not as high as that of other examination tools, such as ultrasonography. Increasingly many physicians are using ultrasonography to evaluate muscle atrophy and fatty infiltration of the supraspinatus muscle, and ultrasound findings were found to be correlated to MRI findings in one study [10]. The primary objective of the present study was to compare the ability of B-mode ultrasonography and MRI to predict the reparability of large-to-massive RCTs. Furthermore, the reliability of B-mode ultrasonography when administered in patients with large-to-massive RCTs was also examined.

\section{Materials and Methods}

\section{Compliance with Ethical Standards}

This study was approved by the Institutional Review Board of Chang Gung Memorial Hospital (IRB No. 201800883BO). A research assistant informed the participants about the study and obtained written informed consent from each patient.

\section{Study Design and Participants}

This was a cross-sectional study conducted at Kaohsiung Chang Gung Memorial Hospital. Participants with large-to-massive RCTs requiring arthroscopic repair were enrolled. Large-to-massive RCTs were defined by the presence of one of two criteria: (1) the diameter of the tear was greater than $3 \mathrm{~cm} \mathrm{[11];} \mathrm{or} \mathrm{(2)} \mathrm{the} \mathrm{full-thickness}$ tear involved two or more tendons [12]. The above criteria were confirmed by MRI or ultrasonography. The exclusion criteria for this study were as follows: (1) severe acromioclavicular arthritis requiring distal clavicle resection; (2) severe glenohumeral arthritis (Hamada classification grade 3 or above) [13]; (3) history of shoulder fracture; and (4) an absolute contraindication for MRI, such as claustrophobia, placement of a cardiac pacemaker, neurostimulator, syringe injection pump, cochlear implant, or any metal implant.

In total, 78 patients with large-to-massive RCTs who planned to undergo arthroscopic repair from October 2018 through September 2020 were screened for participation in this study, and 58 patients provided informed consent and preoperative evaluations were therefore performed. However, eight patients did not ultimately undergo arthroscopic repair, so only 50 patients were included in the final data analysis (Fig. 1).

\section{Data and Assessments}

Demographic characteristics, including age, sex, body height and weight, the side of the lesion, and the duration of symptoms, were recorded at the beginning of the study. The affected shoulder function was evaluated prior to arthroscopic repair: (1) shoulder active range of motion (ROM) at forward flexion, abduction, internal rotation, and external rotation, assessed using a goniometer; and (2) the University of California at Los Angeles shoulder rating scale

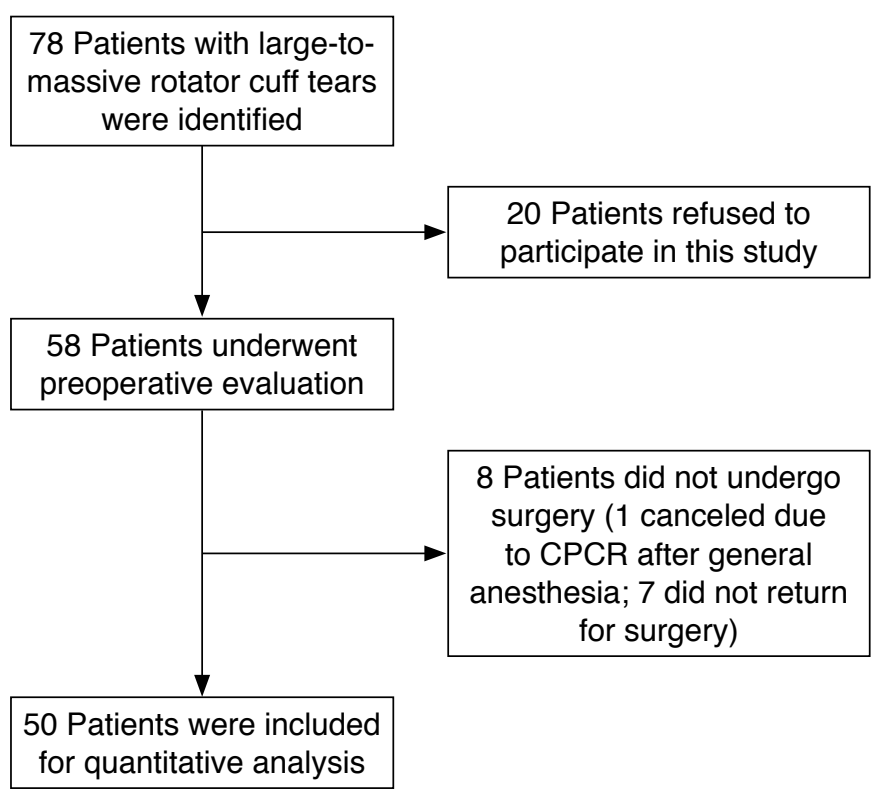

Fig. 1. Flow diagram of the participants. CPCR, cardiopulmonary cerebral resuscitation. 
[11] for a comprehensive assessment of participants' severity of shoulder pain, function, shoulder ROM, shoulder muscle strength, and satisfaction with the surgical outcomes.

All patients were evaluated using both B-mode ultrasonography and MRI prior to surgery. On B-mode ultrasonography, two rotator cuff muscles (the supraspinatus muscle and infraspinatus muscle) were evaluated by physicians with at least 3 years of experience of ultrasonography using a Siemens Acuson S2000 ultrasound system (Siemens Healthcare, Erlangen, Germany), which included a lineararray transducer with a bandwidth of 4-9 MHz. All participants were scanned in a sitting position with a relaxed arm resting on the ipsilateral thigh. The ultrasound transducer was placed perpendicular to the long axis of the supraspinatus and infraspinatus muscle belly, and the region of interest (ROI) was located at the entire middle third part of each muscle. The ultrasound machine was set at the musculoskeletal preset and B-mode with autogain, and the dynamic range was $70 \mathrm{~dB}$ for all participants. Adequate transducer pressure was applied to the skin to obtain optimal images for comparisons of muscle and bone echoes. The Heckmatt scale [12] was utilized to grade the muscle echogenicity within each ROI of the supraspinatus and infraspinatus muscles. Grading was as follows (see Figs. 2 and 3 for grading examples): grade 1, normal; grade 2, increased muscle echo intensity with distinct bone echo; grade 3, markedly increased muscle echo with reduced bone echo; grade 4, very strong muscle echo with complete loss of bone echo. The first 25 of the included 50 participants were selected to test the reliability of the Heckmatt scale within each ROI. Two examiners performed B-mode ultrasonography independently to test the inter-rater reliability. Each examiner also repeated the exam at an interval of 30 minutes to test the intra-rater reliability.

The following MRI images were obtained from a 1.5 T (Signa Horizon LX, GE Healthcare, Chicago, IL, USA) imaging unit equipped with a standard shoulder surface coil. The sequence was performed above the level of the acromioclavicular joint down to below the axillary pouch. The imaging sequences included the following: axial proton-density-weighted fast spin-echo with a fat suppression sequence; coronal oblique proton-density-weighted fast spinecho with and without fat suppression, with the axis parallel to the supraspinatus tendon; and sagittal oblique proton-densityweighted fast spin-echo with and without fat suppression, with the axis perpendicular to the coronal oblique axis. The parameters of fast spin-echo sequences: TR ms/TE ms, 2,700-4,800/25-40; echo-train length, 6; matrix, $256 \times 256$; field of view, $180 \times 180$ $\mathrm{mm}$; section thickness, $2.5 \mathrm{~mm}$ with a $2-\mathrm{mm}$ gap. No intravenous or intra-articular gadolinium was administered. The following MRI parameters were evaluated by a specialist musculoskeletal radiologist: degree of tendon retraction (Patte classification [13]), fatty infiltration of rotator cuff muscles (Goutallier classification [14]), and muscle atrophy (tangent sign [15]) (Supplementary Fig. 1).

\section{Surgical Techniques}

The beach chair position was chosen for all patients. The surgeons first used a $30^{\circ}$ angled arthroscope to build up the posterior entry point for intra-articular exploration. The probe was used to evaluate the biceps brachii tendon, subscapularis tendon, and labrum. An anterior entry point was set up to repair the tendon once any tendon tear was viewed. The arthroscope was switched from the posterior entrance to the acromion after treatment of intra-articular lesions, and the lateral entry point was established for bursectomy. Acromioplasty was performed to remove spurs in the case of any subacromial spurs. The orthopedic surgeons created a posterolateral entry point and converted the arthroscope to this entry point in order to evaluate the rotator cuff lesions. The size of the torn tendons, including the anteroposterior and mediolateral lengths, was measured using a ruler. The orthopedic surgeons released the tendons by electrocautery. To increase the healing ability of the tendon, a curette was used for debridement at the insertion of the rotator cuff tendons with the greater tuberosity. Finally, the orthopedic surgeons placed the affected shoulder at $60^{\circ}$ of abduction for rotator cuff tendon repair. The arthroscopic repair was defined as complete or partial repair according to the intraoperative conditions through discussion between two orthopedic surgeons. A tendon that could be reduced to the inner portion of the footprint was defined as reparable (complete repair); otherwise, it was defined as irreparable (partial repair).

\section{Statistical Methods}

The chi-square test or Fisher exact test was performed to compare the differences in categorical variables between the complete repair and partial repair patient groups. Continuous variables were compared between the two groups using the Mann-Whitney $\mathrm{U}$ test. In order to test the reliability of the measurements on the Heckmatt scale and the hue values of each ROI of the supraspinatus and infraspinatus muscles, the weighted Cohen kappa using average measures was used to evaluate the inter-rater reliability and intrarater reliability. For correlational analysis of the findings of B-mode ultrasonography (Heckmatt scale), MRI findings (Patte classification, Goutallier classification, and tangent sign) and surgical reparability, the Spearman rank correlation was used for continuous variables, while point-biserial correlation was used for comparisons of one continuous variable versus one dichotomous variable. For imaging variables with significant medium or large strengths of association with surgical reparability, a receiver operating characteristic (ROC) curve analysis of the surgical reparability of large-to-massive 
RCTs was conducted to calculate the area under the curve (AUC). A P-value less than 0.05 was defined as indicating statistical significance. The statistical analyses were performed using SAS software version 9.4 (SAS Institute Inc., Cary, NC, USA).

\section{Estimation of Sample Size}

We estimated the sample size for ROC analysis on the basis of a previous study [16]. Approximately half of the large-to-massive RCTs were repaired completely, so the ratio of partial repairs to

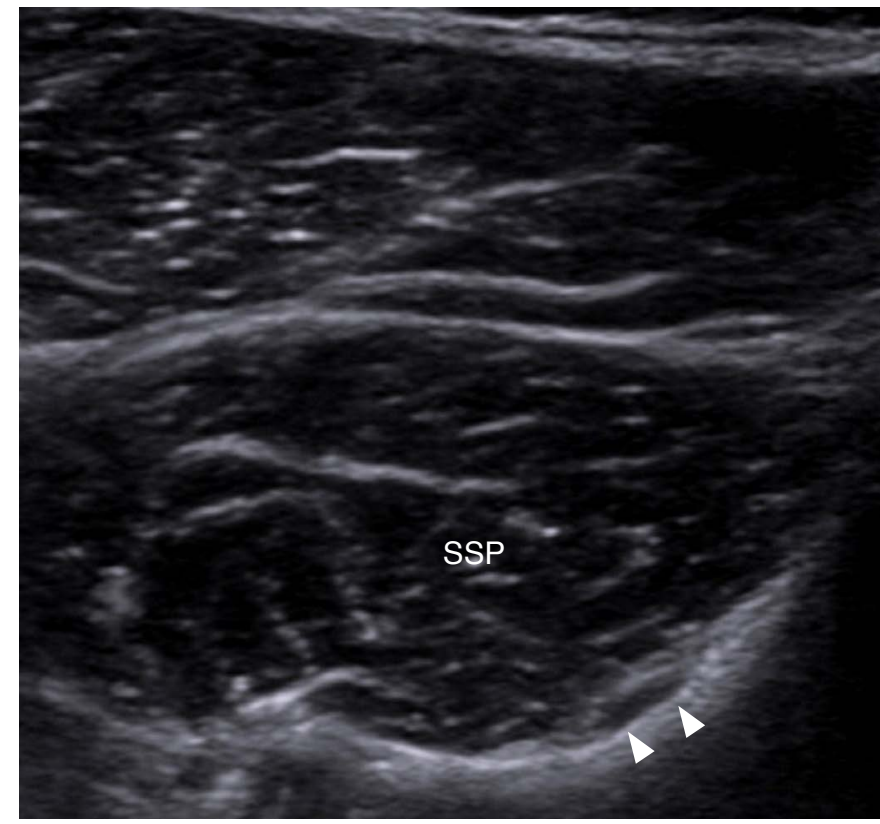

A

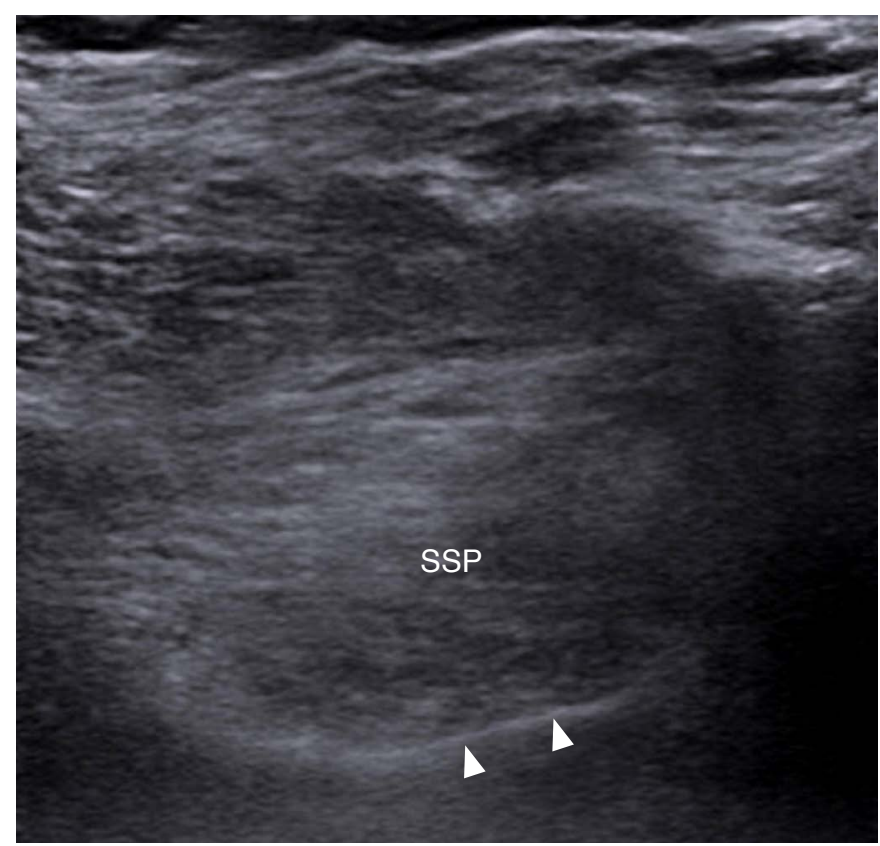

C

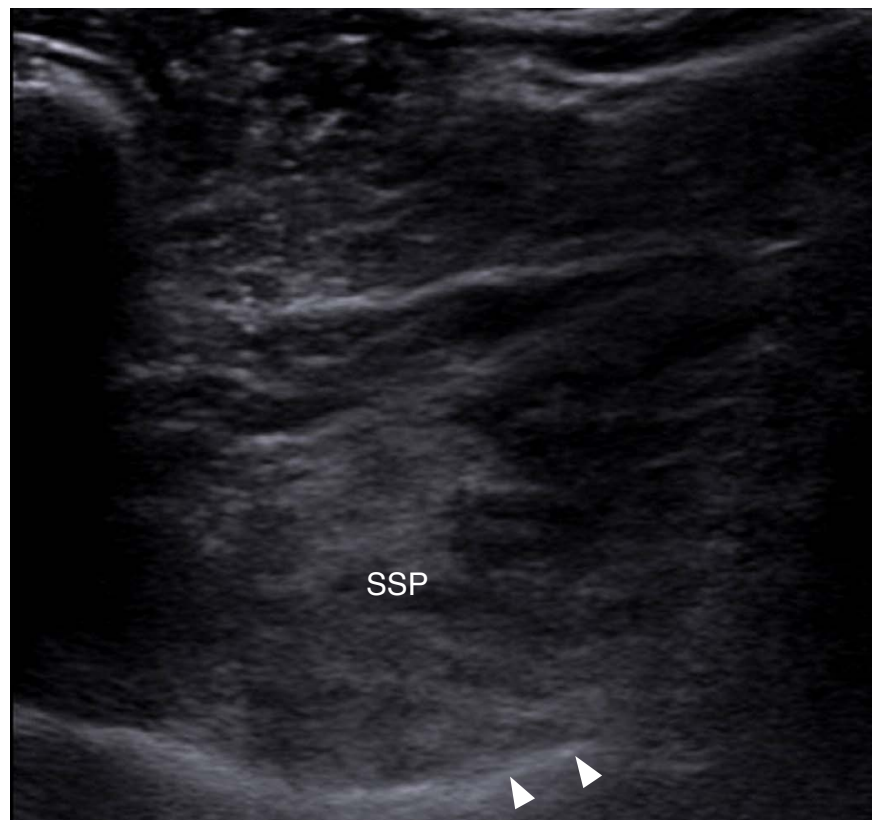

B

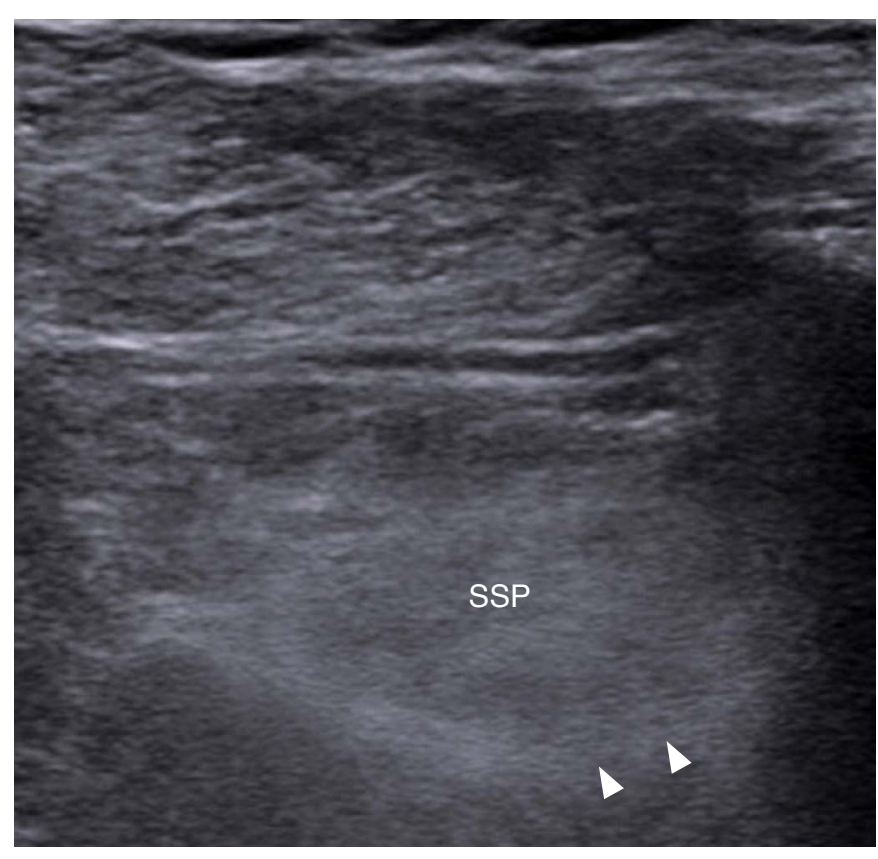

D

Fig. 2. Assessment of the echogenicity of the supraspinatus muscle (SSP) using the Heckmatt scale.

Grading was as follows: grade 1, normal (A); grade 2, increased muscle echo intensity with distinct bone echo (B); grade 3, markedly increased muscle echo with reduced bone echo (C); and grade 4, very strong muscle echo and complete loss of bone echo (D). Arrowheads indicate bone. 
complete repairs was assumed to be 1 [17]. We assumed that (1) type I error=0.05, (2) type II error=0.2, and (3) an acceptable discrimination of 0.7 ; the resulting sample size was calculated to be 62.

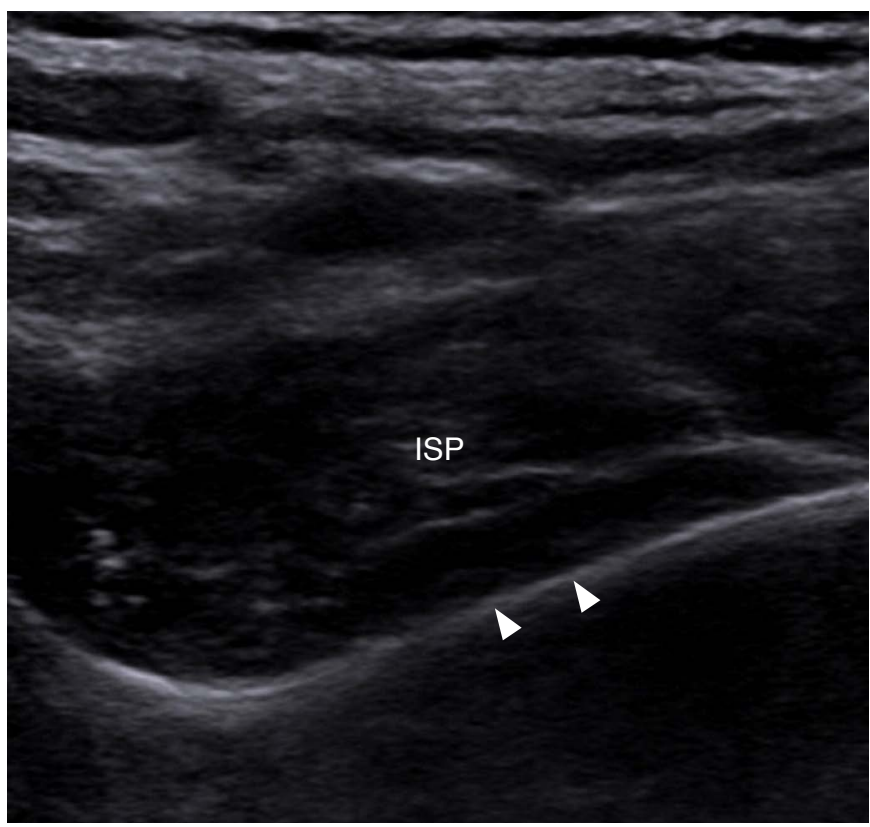

A

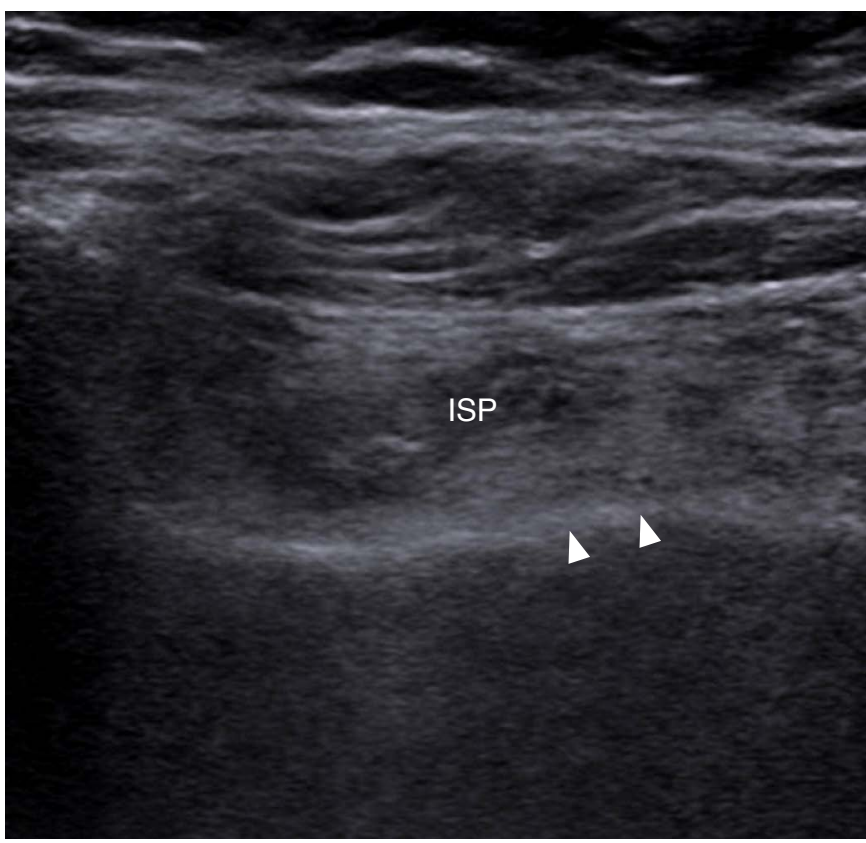

C

\section{Results}

\section{Baseline Characteristics}

There were 32 participants (18 men and 14 women) in the

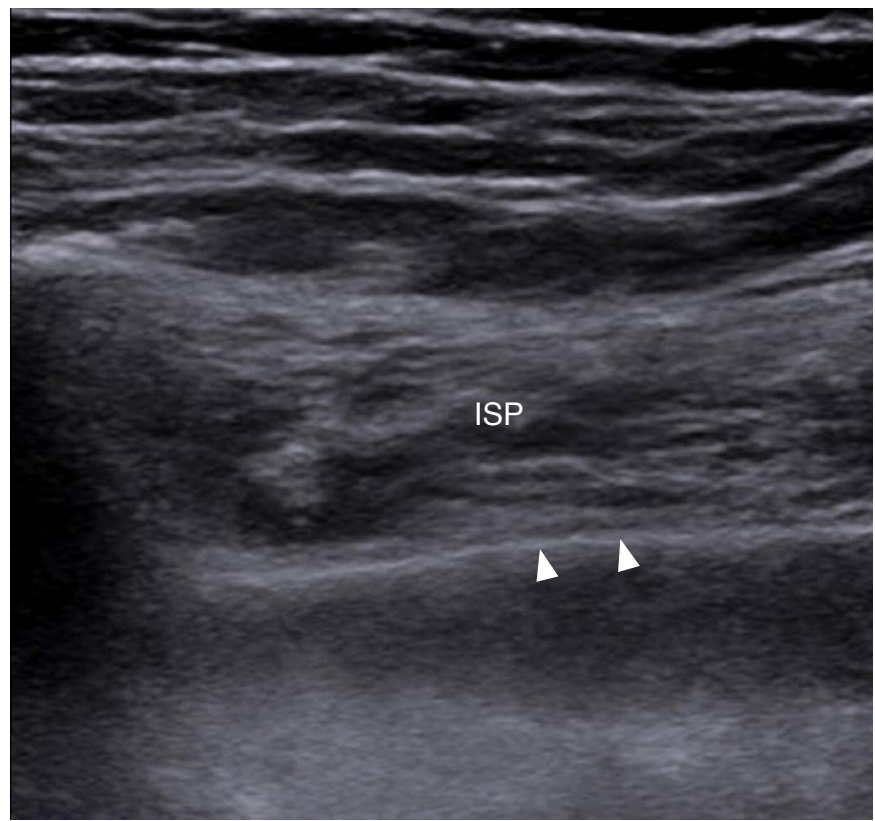

B

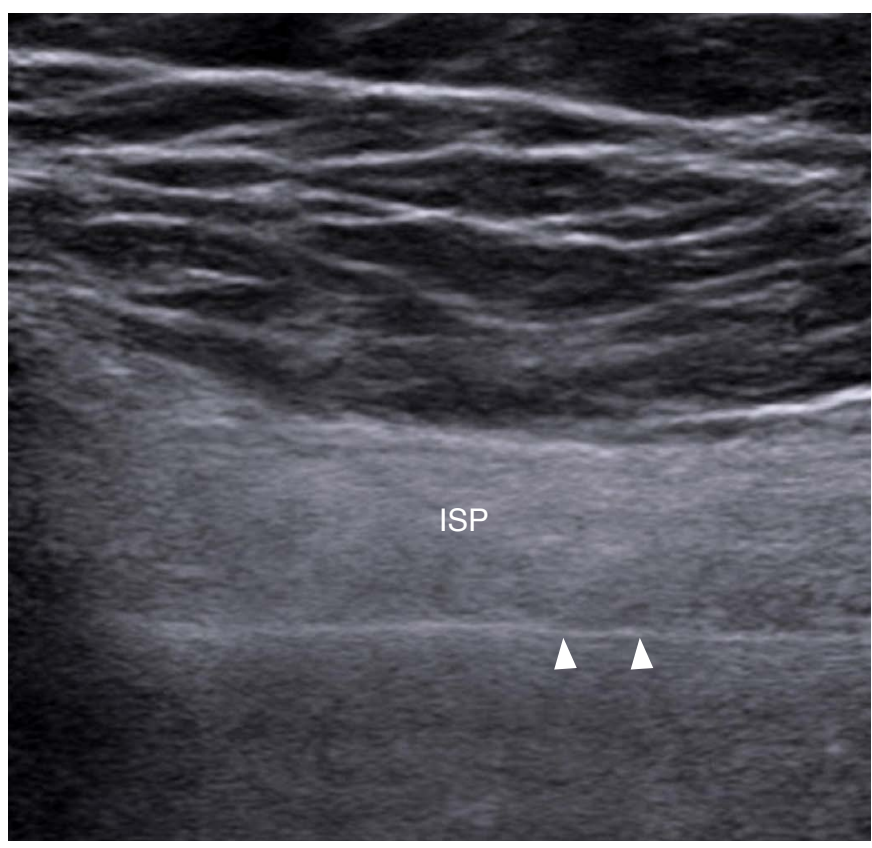

D

Fig. 3. Assessment of the echogenicity of the infraspinatus muscle (ISP) using the Heckmatt scale.

Grading was as follows: grade 1, normal (A); grade 2, increased muscle echo intensity with distinct bone echo (B); grade 3, markedly increased muscle echo with reduced bone echo (C); and grade 4, very strong muscle echo and complete loss of bone echo (D). Arrowheads indicate bone. 
complete repair group and 18 participants (11 men and 7 women) in the partial repair group. The elderly (aged older than 65 years) accounted for 16 out of 32 participants in the complete repair group and 14 out of 18 participants in the partial repair group. No statistically significant differences in the baseline characteristics existed between the complete repair and partial repair groups (Table 1).

\section{Intra-rater and Inter-rater Reliability}

The investigators selected 25 patients to test the reliability of B-mode ultrasonography for assessing the muscle echogenicity of the supraspinatus and infraspinatus muscles. Table 2 shows the intra-rater and inter-rater reliability of B-mode ultrasonography, which were good to excellent for both the supraspinatus and infraspinatus muscles.

\section{Distribution of MRI and B-Mode Ultrasound Parameters}

The percentage of patients with later stage/grades of the Patte classification and Goutallier classification was higher in the partial repair group than in the complete repair group. The percentage of a positive tangent sign of the partial repair group also outweighed that of the complete repair group. Similarly, the percentage of a later grade of the Heckmatt scale of both the supraspinatus and infraspinatus muscles was higher in the partial repair group than in the complete repair group. The distribution of imaging parameters is presented in Table 3.

\section{Predictive Validity of Image Predictors}

The effect sizes of the correlation coefficients between B-mode ultrasound findings and MRI findings were medium to large $(r=0.4-$

Table 1. Baseline characteristics of the participants

\begin{tabular}{|c|c|c|c|}
\hline Characteristic & $\begin{array}{l}\text { Complete repair } \\
\quad(n=32)\end{array}$ & $\begin{array}{l}\text { Partial repair } \\
(n=18)\end{array}$ & P-value \\
\hline Sex (male:female) & $18: 14$ & $11: 7$ & 0.971 \\
\hline Affected side (right:left) & $24: 8$ & $12: 6$ & 0.763 \\
\hline $\begin{array}{l}\text { Duration of symptoms } \\
\text { ( } \leq 12 \text { months: }>12 \text { months) }\end{array}$ & $23: 9$ & $12: 6$ & 0.949 \\
\hline Age ( $\leq 65$ years: $>65$ years) & $16: 16$ & $4: 14$ & 0.074 \\
\hline Age (year), mean (range) & $64.9(53-77)$ & $68.5(60-75)$ & - \\
\hline BMl & $26.08 \pm 4.35$ & $24.58 \pm 2.44$ & 0.221 \\
\hline Flexion ROM & $131.25 \pm 39.02$ & $137.22 \pm 42.19$ & 0.530 \\
\hline Abduction ROM & $120.38 \pm 41.45$ & $125.56 \pm 36.34$ & 0.808 \\
\hline Internal rotation ROM & $61.41 \pm 29.52$ & $61.11 \pm 32.02$ & 0.949 \\
\hline External rotation ROM & $64.38 \pm 28.70$ & $62.22 \pm 30.01$ & 0.924 \\
\hline Preoperative UCLA scale & $19.74 \pm 7.08$ & $20.16 \pm 6.61$ & 0.984 \\
\hline
\end{tabular}

BMI, body mass index; ROM, range of motion; UCLA, University of California at Los Angeles.
0.8) (Table 4). The ROC curves for each MRI and B-mode predictor were plotted, as shown in Fig. 4. The AUC of each image predictor ROC curve is presented in Table 5. Among the MRI predictors, the Goutallier classification of the infraspinatus muscle had the best discriminative power for surgical reparability (AUC, 0.89; $95 \%$ confidence interval $[\mathrm{Cl}], 0.81$ to 0.98 ; sensitivity, 0.67 ; specificity, 0.94 ), with a cutoff value of 2 . Among the ultrasound predictors, the Heckmatt scale for the infraspinatus muscle was the most accurate in terms of predicting surgical reparability (AUC, $0.85 ; 95 \% \mathrm{Cl}, 0.74$ to 0.96 ; sensitivity, 0.67 ; specificity, 0.91 ), with a cutoff value of 3 . However, there were no significant differences in the AUC among the MRI and ultrasound predictors.

We presented the B-mode ultrasound images, MRI, and arthroscopic images together to facilitate better clinical correlation. A case with less fatty infiltrated rotator cuff muscles was completely repaired (Fig. 5), while a case with greater fatty infiltrated rotator cuff muscles was only partially repaired through superior capsular reconstruction (Fig. 6).

\section{Discussion}

The present study evaluated the reliability and validity of B-mode ultrasonography when applied for the preoperative evaluation of large-to-massive RCTs. The intra-rater and inter-rater reliabilities of B-mode ultrasonography were good to excellent for evaluation of the supraspinatus and infraspinatus muscles. The strengths of the correlations among predictors of B-mode ultrasonography and MRI were medium to large. ROC analysis showed that B-mode ultrasonography had as good a predictive validity as MRI for surgical reparability.

Analyses of the association between preoperative MRI evaluation and surgical reparability of large-to-massive RCTs have been conducted in previous studies $[7-9,18,19]$. Jain et al. [20] confirmed the reliability of shoulder MRI assessment of rotator cuff muscles, and high inter-rater and intra-rater reliabilities for most findings on shoulder MRI were observed. The tear size was found to predict

Table 2. Intra-rater reliability and inter-rater reliability of the Heckmatt scale for the supraspinatus and infraspinatus muscles $(\mathrm{n}=25)$

\begin{tabular}{lccc}
\hline & \multicolumn{3}{c}{ Weighted kappa (95\% Cl) } \\
\cline { 2 - 4 } & $\begin{array}{c}\text { Intra-rater } \\
\text { reliability } \\
\text { (examiner A) }\end{array}$ & $\begin{array}{c}\text { Intra-rater } \\
\text { reliability } \\
\text { (examiner B) }\end{array}$ & $\begin{array}{c}\text { Inter-rater } \\
\text { reliability } \\
\text { (A vs. B) }\end{array}$ \\
\hline Supraspinatus muscle & $0.78(0.61-0.94)$ & $0.90(0.79-1.00)$ & $0.84(0.71-0.97)$ \\
Infraspinatus muscle & $0.96(0.89-1.00)$ & $0.93(0.85-1.00)$ & $0.91(0.81-1.00)$ \\
\hline $\mathrm{Cl}$, confidence interval. & & &
\end{tabular}


Table 3. Distribution of MRI and B-mode ultrasound parameters between the complete and partial repair groups

\begin{tabular}{|c|c|c|c|}
\hline MRI parameter & $\begin{array}{c}\text { Complete repair } \\
(n=32)\end{array}$ & $\begin{array}{l}\text { Partial repair } \\
\quad(n=18)\end{array}$ & P-value \\
\hline \multicolumn{4}{|l|}{ Patte classification } \\
\hline Stage 1 & 8 & 0 & 0.001 \\
\hline Stage 2 & 16 & 4 & \\
\hline Stage 3 & 8 & 14 & \\
\hline \multicolumn{4}{|l|}{$\begin{array}{l}\text { Goutallier classification, } \\
\text { supraspinatus }\end{array}$} \\
\hline Grade 0 & 3 & 0 & 0.009 \\
\hline Grade 1 & 5 & 1 & \\
\hline Grade 2 & 15 & 3 & \\
\hline Grade 3 & 8 & 9 & \\
\hline Grade 4 & 1 & 5 & \\
\hline \multicolumn{4}{|l|}{$\begin{array}{l}\text { Goutallier classification, } \\
\text { infraspinatus }\end{array}$} \\
\hline Grade 0 & 7 & 1 & $<0.001$ \\
\hline Grade 1 & 11 & 0 & \\
\hline Grade 2 & 12 & 5 & \\
\hline Grade 3 & 2 & 5 & \\
\hline Grade 4 & 0 & 7 & \\
\hline \multicolumn{4}{|l|}{ Tangent sign } \\
\hline Positive & 10 & 16 & $<0.001$ \\
\hline Negative & 22 & 2 & \\
\hline \multicolumn{4}{|c|}{ B-mode ultrasound parameters } \\
\hline \multicolumn{4}{|c|}{ Heckmatt scale, supraspinatus } \\
\hline Grade 1 & 14 & 2 & 0.002 \\
\hline Grade 2 & 10 & 2 & \\
\hline Grade 3 & 8 & 11 & \\
\hline Grade 4 & 0 & 3 & \\
\hline \multicolumn{4}{|c|}{ Heckmatt scale, infraspinatus } \\
\hline Grade 1 & 14 & 1 & $<0.001$ \\
\hline Grade 2 & 15 & 5 & \\
\hline Grade 3 & 0 & 7 & \\
\hline Grade 4 & 3 & 5 & \\
\hline
\end{tabular}

$\mathrm{MRI}$, magnetic resonance imaging.

the surgical reparability in three studies [7-9], with varying cutoff values of the mediolateral and anteroposterior length. In one study, irreparable tendons were usually seen in cases with a mediallyretracted tendon stump [19]. Fatty infiltration of the supraspinatus and infraspinatus muscles has been confirmed to be an important marker for predicting surgical reparability $[7,19]$. A recent study demonstrated that the Goutallier classification, tangent sign, and Patte classification possessed a superior ability to predict the reparability of massive RCTs [18], and the discrimination of this
Table 4. Correlations between MRI findings and B-mode ultrasound findings in patients with large-to-massive rotator cuff tears $(n=50)$

\begin{tabular}{lcc}
\hline & \multicolumn{2}{c}{ Ultrasound } \\
\cline { 2 - 3 } & $\begin{array}{c}\text { Heckmatt scale, Heckmatt scale, } \\
\text { supraspinatus }\end{array}$ & infraspinatus \\
\hline MRI & 0.46 & 0.51 \\
Patte classification & 0.001 & $<0.001$ \\
P-value & 0.80 & 0.55 \\
Goutallier classification, supraspinatus & $<0.001$ & $<0.001$ \\
P-value & 0.65 & 0.80 \\
Goutallier classification, infraspinatus & $<0.001$ & $<0.001$ \\
P-value & $0.52^{\mathrm{a})}$ & $0.40^{\mathrm{a})}$ \\
Tangent sign & $<0.001$ & 0.004 \\
P-value & &
\end{tabular}

$\mathrm{MRI}$, magnetic resonance imaging.

a)Point-biserial correlation.

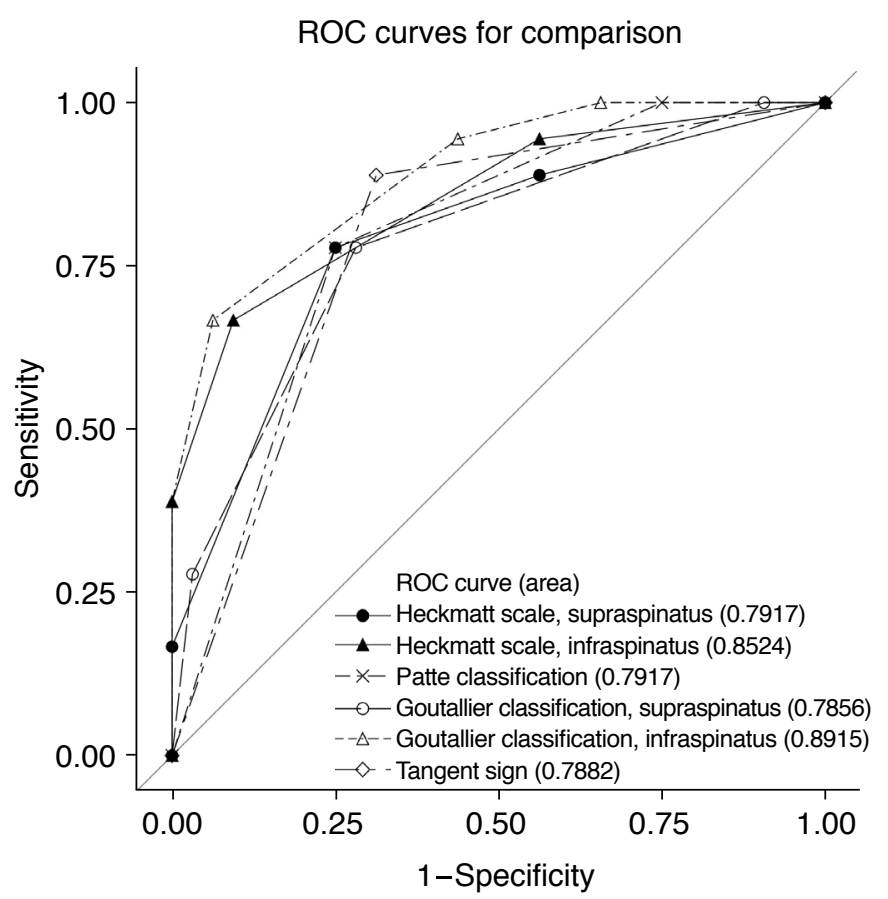

Fig. 4. Receiver operating characteristic (ROC) curves for surgical reparability for different imaging predictors.

model was excellent.

Recently, clinicians have used B-mode ultrasonography to predict the surgical reparability of RCTs, which was found to be related to the size of the tear and patient age [21]. A preliminary report demonstrated the predictive power of B-mode ultrasonography, although the enrolled cases were not specifically limited to largeto-massive RCTs. Khoury et al. [10] found a good correlation 


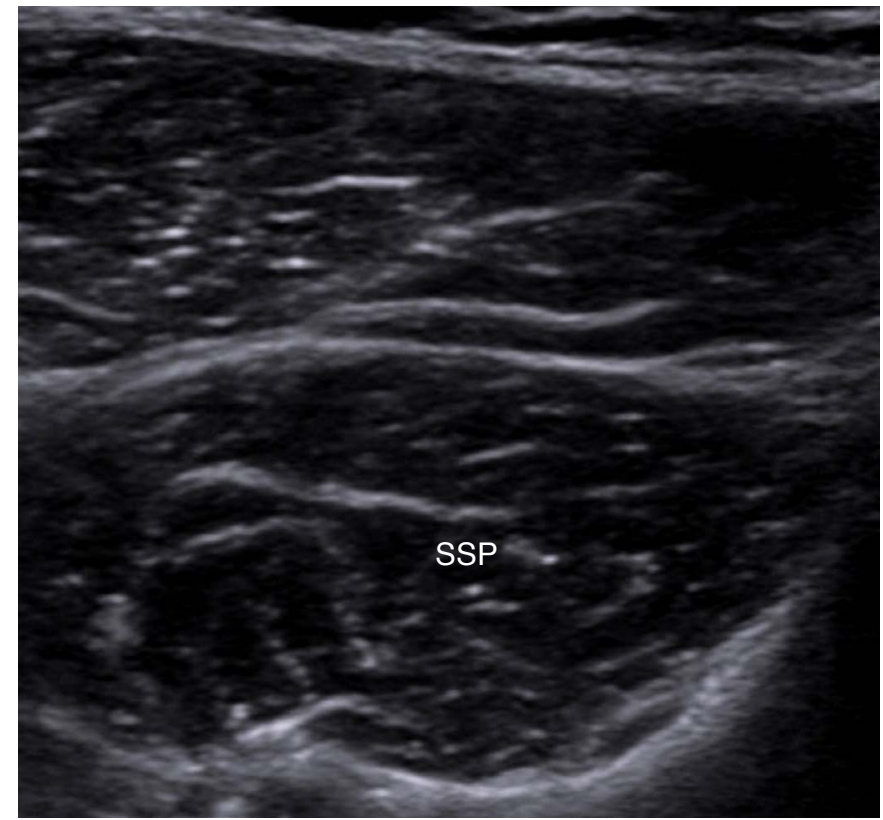

A

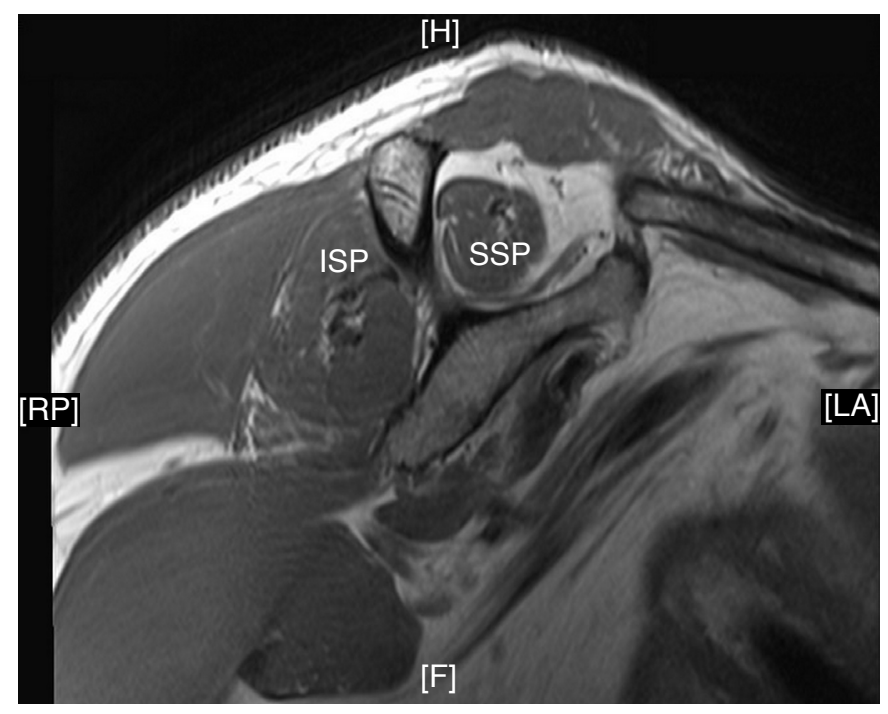

C

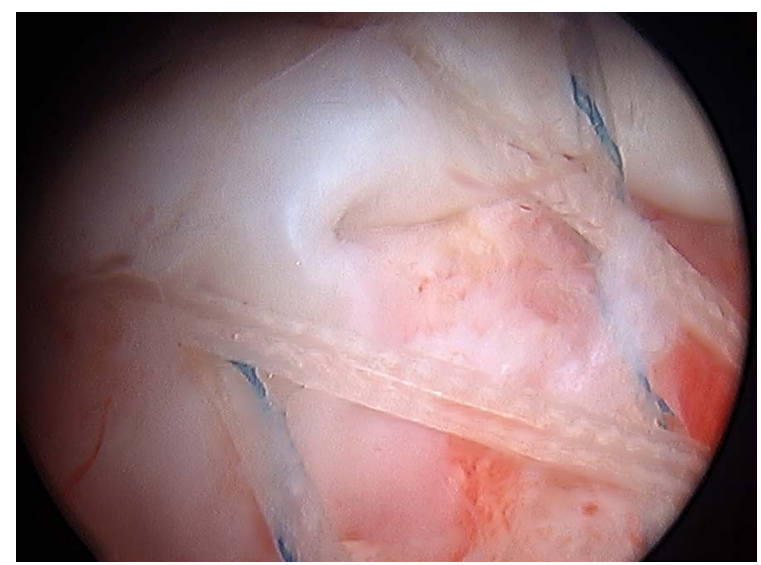

E

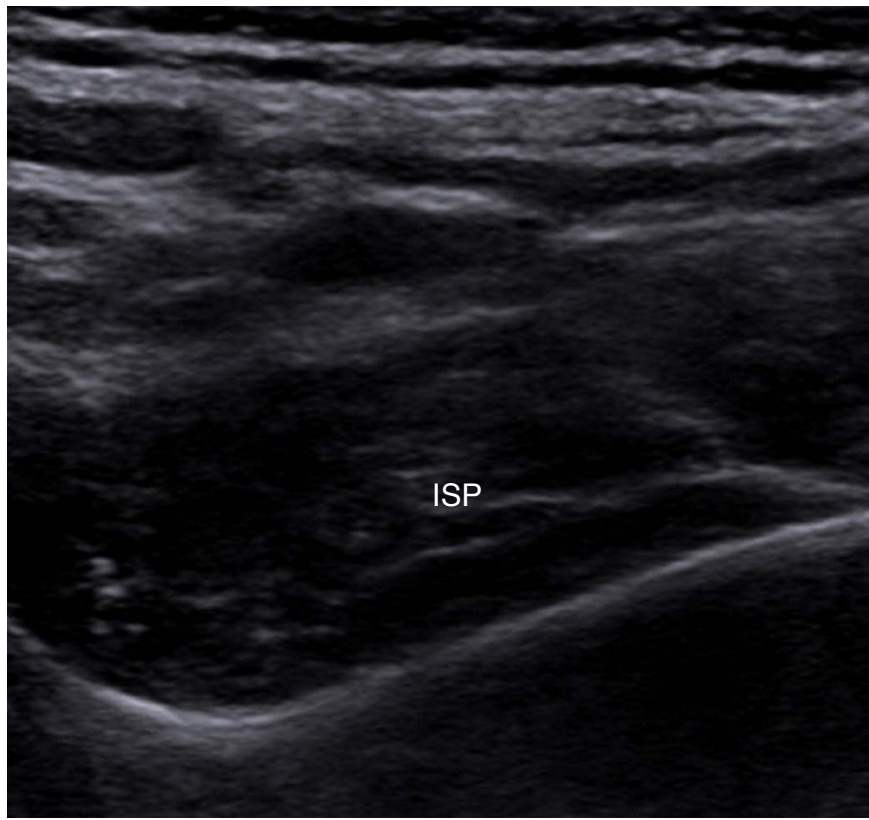

B

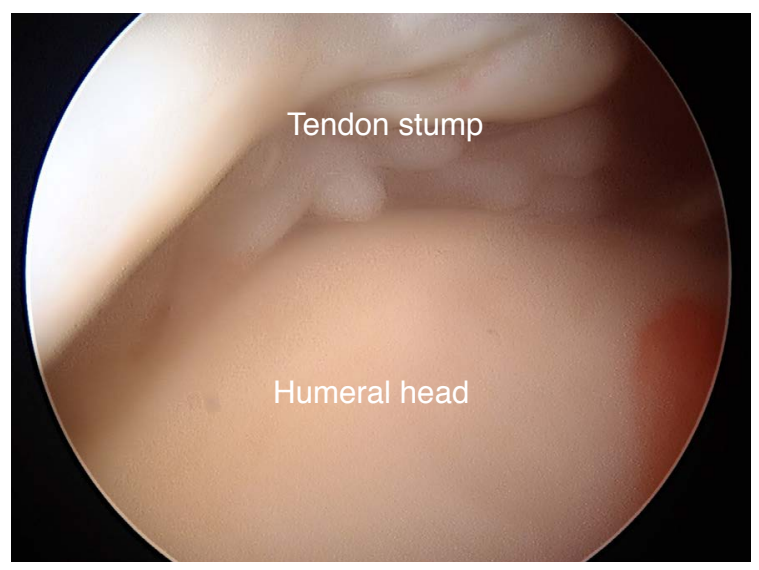

D

Fig. 5. A 57-year-old man with relatively low fatty infiltration of the rotator cuff muscles.

A. B-mode ultrasonography shows the supraspinatus muscle (SSP), Heckmatt scale grade 1. B. B-mode ultrasonography shows the infraspinatus muscle (ISP), Heckmatt scale grade 1. C. Magnetic resonance imaging parameters determined on coronal oblique proton-density-weighted sequences are as follows: Patte classification stage 1, negative tangent sign, Goutallier classification of supraspinatus grade 2, Goutallier classification of infraspinatus grade 1. D. Arthroscopy shows the torn tendon. E. Arthroscopic results show suture bridge repair with double rows for large tear of supraspinatus tendon (tear size: $2 \times 3 \mathrm{~cm}$ ). 


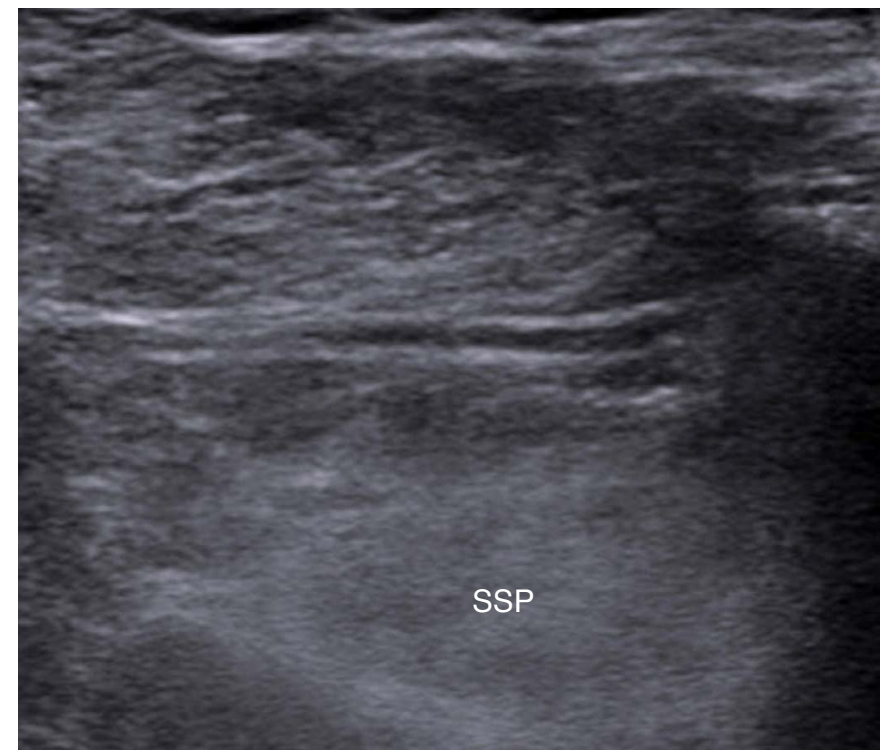

A

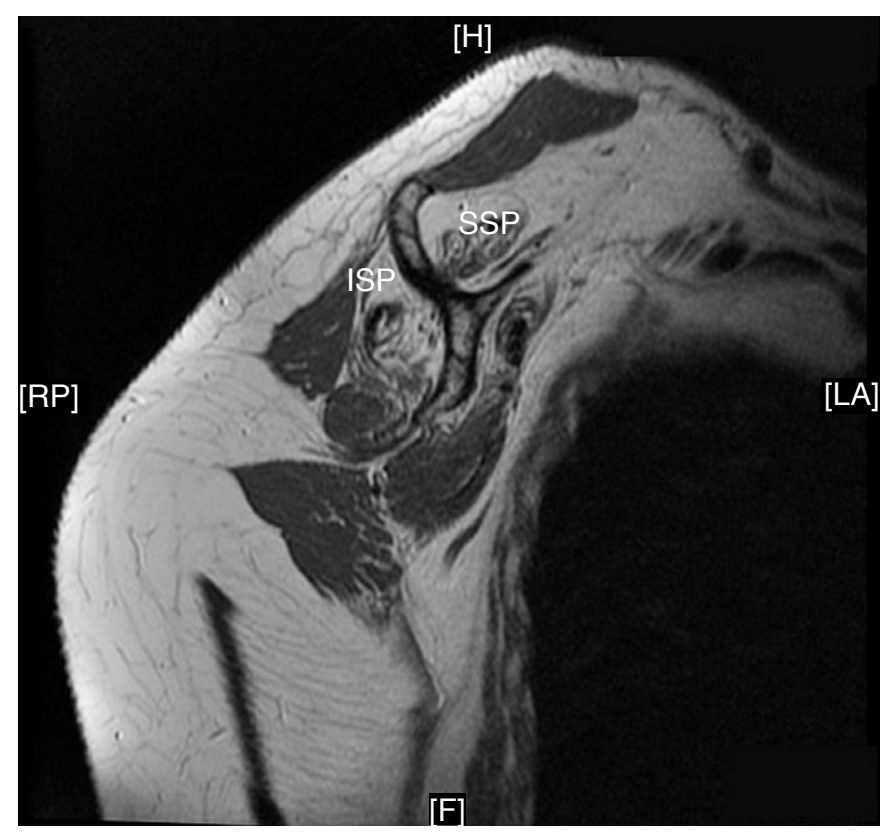

C

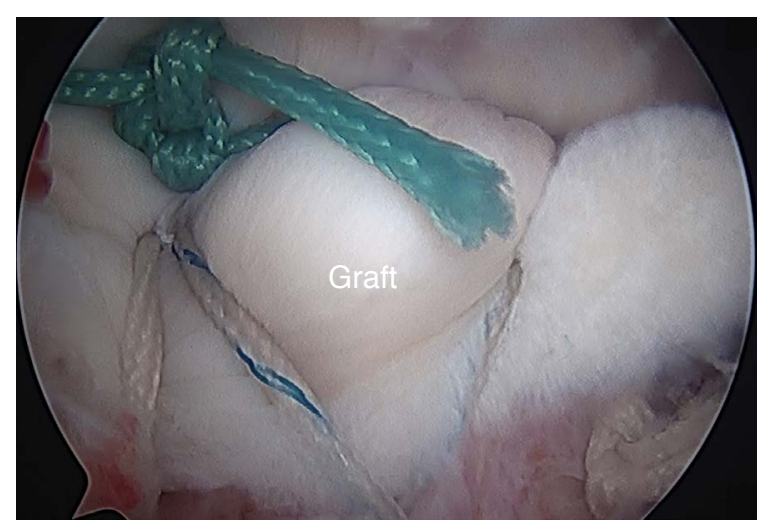

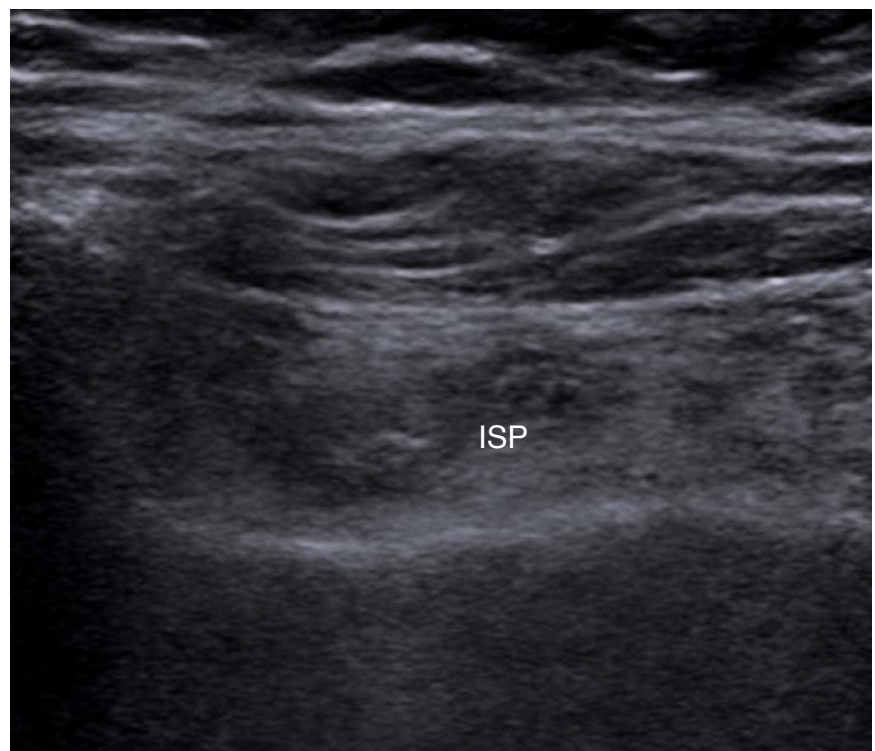

B



D

Fig. 6. A 58-year-old woman with extensive fatty infiltration of the rotator cuff muscles.

A. B-mode ultrasonography shows the supraspinatus muscle (SSP), Heckmatt scale grade 3. B. B-mode ultrasonography shows the infraspinatus muscle (ISP), Heckmatt scale grade 4. C. Magnetic resonance imaging parameters determined on coronal oblique proton-density-weighted sequences are as follows: Patte classification stage 3, positive tangent sign, Goutallier classification of supraspinatus grade 3, Goutallier classification of infraspinatus grade 4. D. Arthroscopy shows the torn tendon. E. Arthroscopic results show superior capsular reconstruction with an acellular dermal allograft for massive tear of the supraspinatus and infraspinatus tendons (tear size: $3.8 \times 3.1 \mathrm{~cm}$ ). 
Table 5. Clinical, MRI, and ultrasound predictors for the ROC models associated with reparability

\begin{tabular}{|c|c|c|c|c|c|}
\hline ROC model & AUC & Wald $95 \% \mathrm{Cl}$ & Sensitivity & Specificity & Cutoff value \\
\hline \multicolumn{6}{|l|}{ MRI predictor } \\
\hline Patte classification & 0.79 & $0.68-0.90$ & 0.78 & 0.75 & $>2$ \\
\hline Goutallier classification, supraspinatus & 0.79 & $0.66-0.91$ & 0.78 & 0.72 & $>2$ \\
\hline Goutallier classification, infraspinatus & 0.89 & $0.81-0.98$ & 0.67 & 0.94 & $>2$ \\
\hline Tangent sign & 0.79 & $0.68-0.90$ & 0.89 & 0.69 & - \\
\hline \multicolumn{6}{|l|}{ Ultrasound predictor } \\
\hline Heckmatt scale, supraspinatus & 0.79 & $0.66-0.92$ & 0.78 & 0.75 & $>2$ \\
\hline Heckmatt scale, infraspinatus & 0.85 & $0.74-0.96$ & 0.67 & 0.91 & $>3$ \\
\hline
\end{tabular}

$\mathrm{MRI}$, magnetic resonance imaging; $\mathrm{ROC}$, receiver operating characteristic; $\mathrm{AUC}$, area under the curve; $\mathrm{Cl}$, confidence interval.

between B-mode ultrasound and MRI findings for the assessment of supraspinatus muscle atrophy and fatty infiltration. However, the echogenicity of the supraspinatus muscles was graded in reference to the surrounding trapezius muscles. The echogenicity of the surrounding bones was less variable than that of the surrounding muscles (trapezius), implying that the surrounding bone might be a good reference for grading the echogenicity of rotator cuff muscles. The muscle echogenicity increased and became similar to the echogenicity of the surrounding bones after the muscle atrophy and fatty infiltration occurred. Heckmatt et al. [12] advocated a grading system to evaluate the intensity of the echo reflected from the muscles. This grading system has been widely used in patients with neuromuscular or upper motor neuron disorders, and its reliability has been examined among patients with cerebral palsy, inclusion body myositis, or spasticity [22-24]; however, it has rarely been applied to evaluate muscle echogenicity in orthopedic patients. Although the patients in our series were quite different from those with neuromuscular or upper motor neuron disorders, muscle atrophy and fatty infiltration of rotator cuff muscles usually occur in the later stages of large-to-massive RCTs, and it was therefore considered reasonable to evaluate the degree of muscular change according to the Heckmatt scale. The investigators in the present study also examined the reliability of the Heckmatt scale for assessing rotator cuff muscles, and found good the intra-rater and inter-rater reliabilities.

Both MRI and B-mode ultrasonography showed higher predictive ability for the infraspinatus muscle (Table 5). A possible explanation for this finding might be that the infraspinatus muscle plays a more important role than the supraspinatus muscle in the surgical reparability of massive RCTs. Furthermore, Kim et al. [18] provided support for this possibility in their study using MRI to predict the surgical reparability of massive RCTs. Another study also confirmed the importance of infraspinatus fatty infiltration in massive RCTs [25]. A higher degree of fatty infiltration of the infraspinatus muscle, rather than the supraspinatus muscle, seems to be the best indicator of poor muscle quality.

To the authors' knowledge, this was the first study to predict the surgical reparability of large-to-massive RCTs using quantitative data from B-mode ultrasonography. B-mode ultrasonography is more available than MRI in clinical settings, and its cost is lower. The reliability of B-mode ultrasonography was found to be good, and the predictive validity of B-mode ultrasonography for the surgical reparability of large-to-massive RCTs was similar to that of MRI.

Nevertheless, this study had some limitations. First, the number of included patients was smaller than the estimated sample size, but a post hoc power analysis of the ROC curve for the Heckmatt scale for the supraspinatus and infraspinatus muscles indicated sufficient results (96.6\% and $98.8 \%$, respectively). Second, the imaging quality of B-mode ultrasonography was operator-dependent and liable to interpretive error. Fortunately, the resolution and imaging quality of B-mode ultrasonography has improved greatly with advances in technology, and therefore the probability of interpretive error has become less and less. Besides, the grading system of the Heckmatt scale is relatively objective and easy for physicians to learn. Third, a preoperative evaluation via B-mode ultrasonography should be performed by specialized physicians, who cannot be easily substituted by other clinical staff. This may be a barrier to further clinical application in some hospitals. Finally, further health economics research should be conducted to prove the costeffectiveness of this analytical method.

In conclusion, B-mode ultrasonography was found to be a reliable examination tool with good intra-rater and inter-rater reliabilities. B-mode ultrasonography had a similar predictive ability of surgical reparability to that of MRI in patients with large-to-massive RCTs.

ORCID: Po-Cheng Chen: https://orcid.org/0000-0002-6386-2850; Kuan-Ting Wu: https://orcid.org/0000-0003-3953-7860; Yi-Cun Chen: https://orcid.org/0000-00025500-9833; Yu-Chi Huang: https://orcid.org/0000-0002-4198-4910; Ching-Di Chang: 
https://orcid.org/0000-0001-6683-0013; Wei-Che Lin: https://orcid.org/0000-00021326-0587; Wen-Yi Chou: https://orcid.org/0000-0002-8400-3457

\section{※uthor affiliations}

'Department of Physical Medicine and Rehabilitation, Kaohsiung Chang Gung Memorial Hospital, Chang Gung University College of Medicine, Kaohsiung; ${ }^{2}$ Department of Public Health, College of Medicine, National Cheng Kung University, Tainan; Departments of ${ }^{3}$ Orthopedic Surgery and ${ }^{4}$ Diagnostic Radiology, Kaohsiung Chang Gung Memorial Hospital, Chang Gung University College of Medicine, Kaohsiung; ${ }^{5}$ Medical Mechatronic Engineering Program, Cheng Shiu University, Kaohsiung, Taiwan

\section{Author Contributions}

Conceptualization: Chen PC, Chen YC, Huang YC, Lin WC. Data acquisition: Chen $P C$, Chang $C D$. Data analysis or interpretation: Chen PC, Wu KT, Chang CD, Chou WY. Drafting of the manuscript: Chen PC. Critical revision of the manuscript: Wu KT, Chen YC, Huang YC, Chang CD, Lin WC, Chou WY. Approval of the final version of the manuscript: all authors.

\section{Conflict of Interest}

No potential conflict of interest relevant to this article was reported.

\section{Acknowledgments}

This study was funded by Chang Gung Memorial Hospital, Taiwan (grant number: CFRPG8H0171, CMRPG8H0811). The funders had no role in the design of the study, data collection, data analysis, interpretation of data, or writing the manuscript.

\section{Supplementary Material}

Supplementary Table 1. Pictures and grade descriptions of each magnetic resonance imaging parameter (https://doi.org/10.14366/ usg.20192).

\section{References}

1. Factor D, Dale B. Current concepts of rotator cuff tendinopathy. Int J Sports Phys Ther 2014;9:274-288.

2. Harris JD, Pedroza A, Jones GL; MOON (Multicenter Orthopedic Outcomes Network) Shoulder Group. Predictors of pain and function in patients with symptomatic, atraumatic full-thickness rotator cuff tears: a time-zero analysis of a prospective patient cohort enrolled in a structured physical therapy program. Am J Sports Med 2012;40:359-366.

3. Harryman DT 2nd, Hettrich CM, Smith KL, Campbell B, Sidles $J A$, Matsen FA 3rd. A prospective multipractice investigation of patients with full-thickness rotator cuff tears: the importance of comorbidities, practice, and other covariables on self-assessed shoulder function and health status. J Bone Joint Surg Am 2003;85:690-696.

4. Rhee YG, Cho NS, Yoo JH. Clinical outcome and repair integrity after rotator cuff repair in patients older than 70 years versus patients younger than 70 years. Arthroscopy 2014;30:546-554.

5. Minagawa H, Yamamoto N, Abe H, Fukuda M, Seki N, Kikuchi K, et al. Prevalence of symptomatic and asymptomatic rotator cuff tears in the general population: From mass-screening in one village. J Orthop 2013;10:8-12.

6. Kuptniratsaikul V, Laohathaimongkol T, Umprai V, Yeekian C, Prasathaporn N. Pre-operative factors correlated with arthroscopic reparability of large-to-massive rotator cuff tears. BMC Musculoskelet Disord 2019;20:111.

7. Yoo JC, Ahn JH, Yang JH, Koh KH, Choi SH, Yoon YC. Correlation of arthroscopic repairability of large to massive rotator cuff tears with preoperative magnetic resonance imaging scans. Arthroscopy 2009;25:573-582.

8. Davidson JF, Burkhart SS, Richards DP, Campbell SE. Use of preoperative magnetic resonance imaging to predict rotator cuff tear pattern and method of repair. Arthroscopy 2005;21:1428.

9. Sugihara T, Nakagawa T, Tsuchiya M, Ishizuki M. Prediction of primary reparability of massive tears of the rotator cuff on preoperative magnetic resonance imaging. J Shoulder Elbow Surg 2003;12:222-225.

10. Khoury V, Cardinal E, Brassard P. Atrophy and fatty infiltration of the supraspinatus muscle: sonography versus MRI. AJR Am J Roentgenol 2008;190:1105-1111.

11. Amstutz HC, Sew Hoy AL, Clarke IC. UCLA anatomic total shoulder arthroplasty. Clin Orthop Relat Res 1981;(155):7-20.

12. Heckmatt JZ, Leeman S, Dubowitz V. Ultrasound imaging in the diagnosis of muscle disease. J Pediatr 1982;101:656-660.

13. Patte D. Classification of rotator cuff lesions. Clin Orthop Relat Res 1990;(254):81-86.

14. Goutallier D, Postel JM, Bernageau J, Lavau L, Voisin MC. Fatty muscle degeneration in cuff ruptures. Pre- and postoperative evaluation by CT scan. Clin Orthop Relat Res 1994;(304):78-83.

15. Zanetti M, Gerber C, Hodler J. Quantitative assessment of the muscles of the rotator cuff with magnetic resonance imaging. Invest Radiol 1998;33:163-170.

16. Hanley JA, McNeil BJ. The meaning and use of the area under a receiver operating characteristic (ROC) curve. Radiology 1982;143:29-36.

17. lagulli ND, Field LD, Hobgood ER, Ramsey JR, Savoie FH 3rd. Comparison of partial versus complete arthroscopic repair of massive rotator cuff tears. Am J Sports Med 2012;40:1022-1026.

18. Kim JY, Park JS, Rhee YG. Can preoperative magnetic resonance imaging predict the reparability of massive rotator cuff tears? Am J 
Sports Med 2017;45:1654-1663.

19. Dwyer T, Razmjou H, Henry P, Gosselin-Fournier S, Holtby R. Association between pre-operative magnetic resonance imaging and reparability of large and massive rotator cuff tears. Knee Surg Sports Traumatol Arthrosc 2015;23:415-422.

20. Jain NB, Collins J, Newman JS, Katz JN, Losina E, Higgins LD. Reliability of magnetic resonance imaging assessment of rotator cuff: the ROW study. PM R 2015;7:245-254.

21. Tse AK, Lam PH, Walton JR, Hackett L, Murrell GA. Ultrasound determination of rotator cuff tear repairability. Shoulder Elbow 2016;8:14-21.

22. Karvelas KR, Xiao T, Langefeld CD, Walker FO, Pathak S, Caress $J B$, et al. Assessing the accuracy of neuromuscular ultrasound for inclusion body myositis. Muscle Nerve 2019;59:478-481.

23. Moreta MC, Fleet A, Reebye R, McKernan G, Berger M, Farag J, et al. Reliability and validity of the modified Heckmatt scale in evaluating muscle changes with ultrasound in spasticity. Arch Rehabil Res Clin Transl 2020;2:100071.

24. Battisti N, Milletti D, Miceli M, Zenesini C, Cersosimo A. Usefulness of a qualitative ultrasound evaluation of the gastrocnemius-soleus complex with the Heckmatt scale for clinical practice in cerebral palsy. Ultrasound Med Biol 2018;44:2548-2555.

25. Kim JR, Cho YS, Ryu KJ, Kim JH. Clinical and radiographic outcomes after arthroscopic repair of massive rotator cuff tears using a suture bridge technique: assessment of repair integrity on magnetic resonance imaging. Am J Sports Med 2012;40:786-793. 